\title{
Una Propuesta de Integración entre Licenciamiento Ambiental y Gestión de Proyectos en la Ciudad de Río de Janeiro-Brasil
}

\author{
José Luiz Fernandes ${ }^{(1)^{*}}$, Eduardo L. Qualharini(2), Daniel R. Nascimento ${ }^{(2)}$ y Andréa S. C. Fernandes ${ }^{(3)}$ \\ (1) Depto. de Ingeniería de Producción - CEFET / RJ, Río de Janeiro, RJ, Brasil. \\ (e-mail: jluizfernandes@gmail.com) \\ (2) Depto. de Construcción Civil - UFRJ, Río de Janeiro, RJ, Brasil. (e-mail: qualharini@poli.ufrj.br; \\ danieln@poli.ufrj.br) \\ (3) Depto. de Ingeniería Civil - CEFET / RJ, Río de Janeiro, RJ, Brasil. \\ (e-mail: andreascunha@gmail.com) \\ * Autor a quien debe ser dirigida la correspondencia.
}

Recibido Oct. 12, 2016; Aceptado Dic. 22, 2016; Versión final Feb. 17, 2017, Publicado Jun. 2017

\begin{abstract}
Resumen
Este artículo presenta una integración del proceso de licenciamiento ambiental aplicada a Río de Janeiro (Brasil) a través del documento de Estudio e Informe de Impacto Ambiental (EIA/RIMA). Se analiza también la estructuración de las recomendaciones sobre prácticas de gestión de proyectos de acuerdo Instituto de Gestión de Proyectos en las etapas de iniciación, planificación, ejecución, control y cierre. Esto se hace con el fin de identificar la eficiencia en los procesos de integración, las partes interesadas, alcance, tiempo, calidad, costo y la comunicación en el plan de gestión de la EIA/RIMA. Se realizó una contextualización de las políticas de licenciamiento ambiental nacional, estatal y local en Brasil, que son cruciales en la elaboración del estudio de impacto ambiental. La integración de la gestión de proyectos y documentos de gestión ambiental contribuye a formar equipos más comprometidos con responsabilidades ambientales presentes en los planes de trabajo para proyectos ambientales.
\end{abstract}

\section{A proposal for the Integration between Environmental Licensing and Project Management in the city of Rio de Janeiro in Brazil}

\begin{abstract}
This paper presents an integration of the process of environmental licensing applied to Rio de Janeiro (Brazil) through the document Study and Report of Environmental Impact (EIA/RIMA). Also, the study analyzes the structuring of the recommendations of project management practices according to the Project Management Institute in the stages of initiation, planning, execution, control and closure. This is done to identify the efficiency in integration processes, stakeholders, scope, time, quality, cost and communication in the document project management plan EIA/RIMA. The contextualization of the national, state and local environmental licensing policies in Brazil was done. These are crucial in the making of the Environmental Impact Study. The integration of project management and environmental management documents contributes to form teams that are more committed with environmental responsibilities present in the work plans of environmental projects.
\end{abstract}




\section{INTRODUCCIÓN}

Las actividades humanas son el resultado de una relación entre el hombre y el medio ambiente en que viven, siendo necesario retirar los recursos naturales del entorno, para los usos más variados. Es en este contexto que surge el otorgamiento de licencias ambientales, con el reto de conciliar la realización de todas las actividades de una manera sostenible.

De acuerdo con MMA (2009) en la década de 1960 el término "medio ambiente" fue utilizado por primera vez - en una reunión del Club de Roma, cuyo objetivo era la reconstrucción de los países en la posguerra. La primera política relacionada al tema que afectó el área del medio ambiente llegó con la creación de la NEPA (National Environmental Policy Act) de 1969, en los Estados Unidos, lo que institucionalizó al año siguiente, el proceso de Evaluación de Impacto Ambiental (EIA) como un instrumento de política ambiental. Como resultado de esta ley hubo la creación de la Agencia Ambiental de Producción de Estados Unidos (Agencia Estatal de Protección al Medio Ambiente de los Estados Unidos) - USEPA y el Consejo de Calidad Ambiental (Council Environmental Quallity) - CEQ.

En 1992 se llevó a cabo en Río de Janeiro, Brasil, la ECO-92, que contó con la participación de 185 gobiernos. Al final de esta conferencia se adoptaron tres instrumentos: La Declaración de Río sobre el Medio Ambiente; la Declaración de principios para el consenso mundial y uso, conservación y desarrollo sostenible de los bosques y el Programa 21. (www.onu.org.br/rio20 /img/2012/01/rio92.pdf)

Según Nakamura (2013), en el año 2002, la Conferencia de Johannesburgo no trajo grandes novedades para el Derecho Ambiental Internacional (DAl), ya que sus actividades se dirigieron a un enfoque de carácter controlador de las acciones aplicadas en Río-92 y en Estocolmo-72. En 2012, en Río de Janeiro, Brasil se llevó a cabo a Río+20, que incluye dos temas específicos: la Economía Verde (EV) y el Marco Institucional para el Desarrollo Sostenible (DEI). El problema hoy en día se agrava debido a que la eficacia de la DAI depende de la interacción coordenada de otros sectores de la política y de la economía, que deben ser alineados para adquirir recursos financieros.

En Colombia, en respuesta a la Conferencia de Estocolmo, se inició el proceso de protección ambiental con la Ley 23 de 1973. Con Constitución política de 1991 se originó la creación de una Política Nacional de Desarrollo (Artículo 339), la cual se promulgaría más adelante y que, con relación al medioambiente, se conocería con el nombre de Ley 99 de 1993. En Chile, en el artículo 15 de la Ley 19300, se le da al responsable de un proyecto, obra o actividad vía libre para contaminar el ambiente y los recursos naturales por 120 días, si el peticionario tiene como pagar una póliza. En el Distrito Federal de México existe la licencia ambiental única y en Honduras, los proyectos se categorizan en 1, 2 y 3, con los de categoría 3 como los de mayor impacto ambiental, a los que se les otorga licencia ambiental; a los de categoría 2, una autorización ambiental y los de categoría 1, una constancia de registro por su bajo impacto ambiental. En España se maneja el concepto de ventanilla única, pero de la protección y conservación de los recursos naturales y el ambiente estos instrumentos políticos dejan ver sus vacíos (Garzón (2006).

De acuerdo con Verocai (2010) en Chile en 1993, los procedimientos de evaluación ambiental para proyectos comienzan a ser implementados con voluntad a causa de los requisitos del gobierno. Pero, en 1996 se introdujo la declaración y el estudio de impacto ambiental. Estos documentos ayudaron a simplificar la concesión de licencias y aprobación de proyectos. En Argentina, en provincias como Córdoba, Mendoza y Río Negro, la autoridad responsable es el Consejo de Defensa del Medio Ambiente (CODEMA). En Bolivia la Ley 1995 (Ley General de Gestión del Medio Ambiente) es bastante amplia y abarca toda la gama de instrumentos de política y gestión ambiental, allí se requiere el Licenciamiento Ambiental para todas las actividades que modifican el entorno, las mismas tienen una caducidad de 10 años y la participación pública está garantizada para el acceso a los estudios y audiencia pública.

Las Licencias ambientales son propuestas en la Política Nacional de Medio Ambiente de Brasil (Ley $\mathrm{N}^{\circ} 6.938$ / 81), es una herramienta de gestión de los órganos competentes de las medidas preventivas para la protección del medio ambiente. En el proceso de licenciamiento ambiental, las agencias ambientales evalúan los impactos que cada proyecto puede causar al medioambiente mediante el análisis de su viabilidad ambiental y de sus normas ambientales marco. El proceso de obtención de licencias ambientales en Brasil es complejo y difícil para los gestores ambientales y gestores de proyectos. La Tabla 1 muestra las leyes que abordan el tema de la licencia ambiental y que se encuentren vigentes en el municipio de Río de Janeiro (Matos, 2015). 
Tabla 1: Las leyes que tratan de la obtención de licencias ambientales

\begin{tabular}{|c|c|c|}
\hline Legislación & Nivel & Descripción \\
\hline $\begin{array}{l}\text { Constitución Federal } \\
\text { del } 5 \text { de octubre de } \\
1988\end{array}$ & Federal & - \\
\hline $\begin{array}{l}\text { Ley } 6938 \text { del } \\
31 / 08 / 1981 \text { (Política } \\
\text { Ambiental Nacional) }\end{array}$ & Federal & $\begin{array}{l}\text { La Política Nacional de Medioambiente, sus efectos y mecanismos de } \\
\text { formulación y aplicación, y otras medidas }\end{array}$ \\
\hline $\begin{array}{l}\text { Ley de } 7804 \text { del } \\
18 / 07 / 1989\end{array}$ & Federal & $\begin{array}{l}\text { Se modifica la Ley № } 6938 \text {, que establece la Política Nacional del } \\
\text { Medioambiente, sus efectos y mecanismos de formulación y aplicación de [...], y } \\
\text { otras disposiciones }\end{array}$ \\
\hline $\begin{array}{l}\text { Resolución } \\
\text { CONAMA } 001 \text { del } \\
\text { 23/01/1986 }\end{array}$ & Federal & $\begin{array}{l}\text { Establece definiciones, responsabilidades, criterios básicos y directrices } \\
\text { generales para el uso y la aplicación de la Evaluación de Impacto Ambiental }\end{array}$ \\
\hline $\begin{array}{l}\text { Decreto } 99.274 \text { del } \\
\text { 06/06/1990 }\end{array}$ & Federal & Regula la Política Nacional Ambiental \\
\hline $\begin{array}{l}\text { Resolución } \\
\text { CONAMA } 237 \text { del } \\
\text { 19/12/1997 }\end{array}$ & Federal & $\begin{array}{l}\text { Prevé la revisión y cumplimiento de los procedimientos y criterios utilizados para } \\
\text { la concesión de licencias ambientales }\end{array}$ \\
\hline $\begin{array}{l}\text { Ley } 9.605 \mathrm{del} \\
12 / 02 / 1998\end{array}$ & Federal & $\begin{array}{l}\text { Prevé sanciones penales y administrativas derivadas de las conductas y } \\
\text { actividades dañinas para el medioambiente, y otras disposiciones. }\end{array}$ \\
\hline $\begin{array}{l}\text { Decreto } 6.514 \text { del } \\
22 / 07 / 2008\end{array}$ & Federal & $\begin{array}{l}\text { Prevé infracciones y sanciones administrativas para el medio ambiente, } \\
\text { establece el proceso administrativo federal para la investigación de esos delitos, } \\
\text { y otros asuntos. }\end{array}$ \\
\hline $\begin{array}{l}\text { Ley Complementaria } \\
\text { № } 140 \text { del } \\
08 / 12 / 2011\end{array}$ & Federal & $\begin{array}{l}\text { Reglas fijas, [...] para la cooperación entre la Unión, los estados, el Distrito } \\
\text { Federal y los municipios en los procedimientos administrativos derivados del } \\
\text { ejercicio de la responsabilidad común para la protección de los paisajes naturales } \\
\text { excepcionales, a la protección del medio ambiente, la lucha contra la } \\
\text { contaminación en cualquiera de sus formas y la preservación de los bosques, la } \\
\text { fauna y la flora; [...]. }\end{array}$ \\
\hline $\begin{array}{l}\text { Decreto } 8.437 \text { del } \\
22 / 04 / 2015\end{array}$ & Federal & $\begin{array}{l}\text { Regula los dispuesto en el art. } 7 \text {, caput, fracción XIV, letra "h", y el párrafo único } \\
\text { de la Ley Complementaria № } 140 \text {, de } 8 \text { de diciembre de } 2011 \text {, para establecer } \\
\text { los tipos de proyectos y actividades cuya licencia ambiental será competencia de } \\
\text { la Unión }\end{array}$ \\
\hline $\begin{array}{l}\text { Ley } 3.467 \mathrm{del} \\
14 / 09 / 2000\end{array}$ & Estado & $\begin{array}{l}\text { Se prevé sanciones administrativas derivadas de la conducta perjudicial para el } \\
\text { medioambiente en el Estado de Río de Janeiro, y otras medidas. }\end{array}$ \\
\hline $\begin{array}{l}\text { Decreto } 42.050 \text { del } \\
25 / 09 / 2009\end{array}$ & Estado & $\begin{array}{l}\text { Trata de la disciplina de la concesión de licencias procedimiento de la } \\
\text { descentralización del medioambiente mediante la celebración de acuerdos con } \\
\text { los municipios del estado de Río de Janeiro, y otras medidas. }\end{array}$ \\
\hline $\begin{array}{l}\text { Resolución Conema } \\
42 \text { del 17/08/2012 }\end{array}$ & Estado & $\begin{array}{l}\text { Regula las actividades que causan o pueden causar impacto ambiental local y } \\
\text { establece normas generales de la cooperación federativa en procedimientos } \\
\text { administrativos provenientes del ejercicio de la responsabilidad común para la } \\
\text { protección de los paisajes naturales excepcionales, la protección del } \\
\text { medioambiente y lucha contra la contaminación en cualquiera de sus formas. }\end{array}$ \\
\hline $\begin{array}{l}\text { Decreto } 42.440 \text { del } \\
30 / 04 / 2010\end{array}$ & Estado & $\begin{array}{l}\text { Modifica el Decreto } 42050 \text {, que regula la descentralización del procedimiento de } \\
\text { autorización ambiental mediante la celebración de acuerdos con los municipios } \\
\text { del estado de Río de Janeiro, y otras medidas. }\end{array}$ \\
\hline $\begin{array}{l}\text { Decreto } 44.820 \text { del } \\
02 / 06 / 2014\end{array}$ & Estado & Regula el Sistema de Licencias Ambientales - SLAM y otras medidas. \\
\hline $\begin{array}{l}\text { Decreto № } 40.722 \\
\text { del 08/10/2015 }\end{array}$ & Municipal & Regula los procedimientos para el Sistema de Licencias Ambiental Municipal. \\
\hline
\end{tabular}

Con la promulgación del Decreto-Ley №134 del 16 de junio de 1975, se estableció en Río de Janeiro, el sistema de licencias de actividades contaminantes, lo que obliga a la concesión de licencias a nuevas empresas. Por el Decreto № 8468 de 1976 se reglamentó la Ley n ${ }^{\circ}$ 997/76 y se estableció la Sociedad Estatal para el Saneamiento Básico y Tecnología de Defensa del Medio Ambiente - CETESB, como agencia delegada al gobierno de São Paulo responsable por el control, inspección, monitoreo y actividades de 
concesión de licencias de generación de contaminantes, con la preocupación fundamental de preservar y de restaurar la calidad del agua, aire y suelo.

Según Juras (2013) se entiende que la legislación ambiental ha sido apuntada por algunos sectores como un obstáculo para el crecimiento económico del país, siendo que uno de los instrumentos que es objeto de crítica, es la evaluación del impacto ambiental, requisito para la licencia ambiental de obras y actividades, y considerado obstáculo burocrático para la ejecución de proyectos de infraestructura. Se sabe, sin embargo, que Brasil no está aislado en este tema, ya que muchos países tienen reglas para que el crecimiento económico ocurra con respeto al medioambiente como se ha visto anteriormente.

\section{METODOLOGÍA}

En este artículo se presenta un estudio sistemático de todas las leyes y reglamentos relativos a la concesión de licencias ambientales para el estado de Río de Janeiro (Brasil), presenta asi mismo, las etapas del licenciamiento ambiental que se pueden aplicar a otras localidades / países, utilizando las buenas prácticas de gestión de proyectos para la obtención de licencias ambientales, de acuerdo con PMI.

\section{El proceso de licencias ambientales}

Para el desarrollo del proceso de licenciamiento ambiental - LA se requieren varios documentos técnicos proporcionados por el órgano ambiental con la participación del promotor del proyecto, es necesario para iniciar el proceso de concesión de licencias en base a acuerdos de licencia y a sus pasos. Los principales documentos técnicos se describen a continuación: (Nascimento, 2015):

1. Hoja de Caracterización del Proyecto: presenta los principales elementos que caracterizan el proyecto, el porte, la tecnología, la ubicación y principales aspectos ambientales involucrados. Después, el Instituto Nacional de Medio Ambiente y Recursos Naturales Renovables - IBAMA y otras autoridades ambientales competentes identificarán los criterios de responsabilidad de los estudios ambientales detallados necesarios para que se desarrollen los procedimientos específicos para las licencias ambientales, observadas la naturaleza, características peculiaridades de la actividad; e irá armonizar el proceso de concesión de licencias a través de las etapas de planificación, implementación y operación de estas actividades.

2. Términos de referencia: tiene por objeto establecer las directrices para el estudio del medioambiente y es el instrumento rector para su desarrollo, expedida a modo de licencia previa cuando la solicitud de licencia. Los términos de referencia son un paso clave para que la Evaluación de Impacto Ambiental - EIA alcance el fin deseado y la calidad esperada.

3. Evaluación de Impacto Ambiental - EIA y el Informe de Impacto Ambiental - RIMA: Resolución CONAMA №237/97 (2015) también determina que "los estudios necesarios para el proceso de licenciamiento ambiental deben ser realizadas por profesionales legalmente habilitados y a expensas del empresario" y que "el empresario y los profesionales que se suscriben los estudios mencionados en este artículo serán responsables por la información presentada, sin perjuicio de la responsabilidad administrativa, civil y penal" (artículo 11 y párrafo único). En relación a plazos, referentes a los análisis y a la formulación de requisitos adicionales, el órgano ambiental podrá establecer plazos para análisis diferentes, para cada modo de licencia: Licencia Preliminar - LP; Licencia de Instalación y Funcionamiento - LI; Licencia de Operación - LO) siempre que cumplan el periodo máximo de doce (12) meses desde el acto de presentación de la solicitud hasta su aprobación o rechazo. (Resolución CONAMA № 237/97 art.14 de 2015).

4. Informe Ambiental Simplificado - RAS: Ante la necesidad de establecer procedimientos simplificados para la autorización ambiental de proyectos con impacto ambiental de menor alcance, necesarios para mejorar el suministro de electricidad, y en cumplimiento a la Medida Provisional ํㅡ 2152, de 1을 de Junio 2001, el CONAMA, a través de la Resolución CONAMA n²79/01(2015), estableció el Informe Ambiental simplificado - RAS para: centrales hidroeléctricas y sistemas asociados; centrales termoeléctricas y sistemas asociados; sistemas de transmisión de energía eléctrica (líneas de transmisión y subestaciones); y para usinas eólicas y con otras fuentes alternativas de energía. El informe ambiental simplificado consiste en estudios sobre los aspectos medioambientales relacionados con la ubicación, instalación, operación y expansión de una actividad o de un proyecto, presentados como subvenciones para la concesión de la licencia preliminar, que contiene las informaciones relativas a la evaluación ambiental de la región de inserción del proyecto, su caracterización, la identificación de los impactos ambientales y las medidas de control pertinentes.

5. Informe de Control Ambiental - RCA: consiste en estudios sobre los aspectos medioambientales relacionados con la ubicación, instalación, operación y expansión de la actividad o de un proyecto que no genera impactos ambientales significativos, y que contiene información sobre: la caracterización del entorno 
en que se planea instalar; su ubicación frente al Plan Maestro Municipal; permisos y documentos similares; el plan de control ambiental, identificando las fuentes de contaminación o degradación, y las medidas de control pertinentes.

6. Proyecto Básico Ambiental - PBA: es el documento que se presenta, en detalle, todas las medidas de control y programas ambientales propuestos en el Estudio de Impacto Ambiental - EIA. Debe ser presentado para la obtención de la Licencia de Instalación - LI.

7. Plan de Control Ambiental - PCA: debe contener los proyectos ejecutivos de minimización de los impactos ambientales evaluados a través del documento de EIA / RIMA y entregados para la obtención de la Licencia Previa - LP.

8. Informe Detallado de Programas Ambientales - RDPA: presenta, en detalle, todas las medidas de control y los programas ambientales propuestos en el Informe Simplificado Ambiental - RAS, y debe ser presentado junto con la prueba de cumplimiento de las condiciones del LP, al IBAMA y a otras autoridades ambientales competentes, en la aplicación de LI. Así Como el RAS, el informe RDPA se utiliza sólo para proyectos de impacto ambiental de pequeño alcance, lo que es definido por IBAMA y otras autoridades ambientales.

9. Plan de Recuperación de Áreas Degradadas- PRAD: se utiliza, generalmente, para la recuperación de áreas degradadas por la actividad minera. Se puede pedir para regularizar obras no autorizadas o sumadas al Plan de Control Ambiental, la expedición del LI o LO.

10. Estudio de Viabilidad Ambiental - EVA: la Resolución CONAMA №23 del 7 de diciembre de 1994 (CONAMA, 2015), fijó criterios específicos relacionados con la explotación de los combustibles líquidos y los depósitos de gas natural. En este procedimiento hay la concesión de la licencia de producción para la Investigación Preliminar - LPPRO, que autoriza la producción para investigar la viabilidad económica del yacimiento, el empresario debe presentar para la concesión de este acto, la viabilidad ambiental del estudio - EVA, que contiene el plan de desarrollo de producción para la investigación prevista, con la evaluación ambiental y la indicación de las medidas de control que deben adoptarse. La fase de viabilidad tiene un carácter técnico y económico. Su objetivo es obtener, en general, el diseño total de un proyecto dado y sus limitaciones técnicas y evaluar sus mejores alternativas tanto en el aspecto técnico como económico, con el fin de demostrar la viabilidad del proyecto.

11. Informe de Desempeño Ambiental del Proyecto - RPA: para la renovación de la LO, el empresario debe demostrar que el proyecto cumple con todos los requisitos legales y con los compromisos contraídos en las diversas etapas de licenciamiento ambiental. El informe que se presentará al IBAMA y a otras autoridades ambientales deben referirse a todos los elementos que figuran como medidas de mitigación y a la situación en que se encuentran los programas ambientales presentados en la Evaluación de Impacto Ambiental (EIA) y en el Proyecto Básico Ambiental (PBA), así como hacer referencia a todos los elementos adicionales que se han incorporado en el informe de aplicación de los programas de medioambiente y el cumplimiento de las eventuales condiciones para la operación del proyecto, requerido por el IBAMA y por otras autoridades ambientales en el momento de la obtención de la LO. También se debe describir el marco de gestión ambiental pensada por el empresario.

\section{Pasos de concesión de licencias}

La licencia ambiental en la ciudad de Río de Janeiro (Brasil) puede ser dividida por lo menos en once pasos como se muestra en la Figura 1. La agencia ambiental, después de un examen previo de la documentación, presenta el informe técnico que define si se somete al estudio ambiental. En caso afirmativo, se deben presentar los siguientes documentos: Informe de Estudio de Impacto Ambiental / Evaluación de Impacto Ambiental (EIA / RIMA), informe antes del impacto (RIAP), informe de impacto en el barrio (RIV), informe de impacto Ambiental Suplementario (RIAC), entre otros. En esta etapa, los técnicos de las agencias ambientales llevan a cabo la inspección en el lugar que se verán afectados.

En la expedición de los Términos de Referencia, la agencia ambiental sólo define las directrices después de consultar a otros organismos que incluirán sus manifestaciones y contribuciones. La preparación de los Términos de Referencia es uno de los temas críticos del proceso de licenciamiento ambiental. No siempre las agencias ambientales tienen modelos específicos de Términos de Referencia para cada tipo de actividad ambiental para obtener una licencia. El empresario, teniendo el Término de Referencia, prepara el estudio definitivo. Si el estudio ambiental es una EIA / RIMA, estos deberán estar disponibles para consulta pública en lugares fácilmente accesibles a la población, se establece un plazo máximo de 45 días para manifestación y comentarios. 
La agencia ambiental fija sobre aviso y anuncia en la Gaceta Oficial (DOU) haber recibido la evaluación ambiental (EIA / RIMA) y establece una fecha límite para solicitar una audiencia pública, por entidad, el Ministerio Público, por cincuenta o más ciudadanos. En algunos estados de Brasil, una audiencia pública es obligatoria. Las expresiones de interés en las audiencias públicas que implican que la agencia ambiental pida al empresario más aclaraciones e información adicional sobre los estudios que podrían dar lugar a cambios en los proyectos y cambios en su ubicación. El órgano ambiental recibe el estudio ambiental elaborado por el empresario y forma comité técnico para el análisis del estudio. Este comité puede tener técnicos de otros organismos implicados en la concesión de licencias. El cuerpo técnico presenta el parecer técnico del análisis ambiental, aprobando integralmente o solicitando aclaraciones y / o adiciones en el estudio.

Con la finalización del análisis técnico y teniendo del informe técnico, la agencia ambiental emite o no el LP. La LP emitida tiene una caducidad no superior a cinco años, el empresario deberá cumplir con todos los requisitos solicitados antes de la caducidad o cuando se entregue la solicitud LI.

De acuerdo con la Resolución CONAMA № 237/1997 (2015), la autoridad ambiental competente podrá establecer periodos de análisis diferenciados para cada modo de licencia (LP, LI y LO), en función de las peculiaridades de la actividad o proyecto, así como para la formulación de requisitos adicionales, teniendo debidamente en cuenta el período máximo de seis meses después del acto de presentación de la solicitud hasta su aprobación o rechazo, excepto en los casos en que existe EIA / RIMA y/o audiencia pública, cuando el plazo será de doce meses.
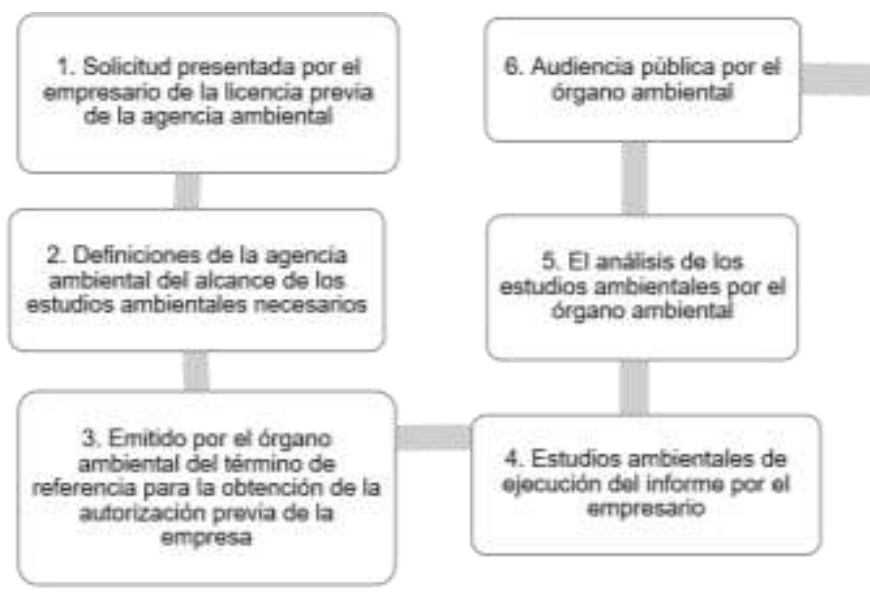

Fig. 1: Etapas de Licenciamiento Ambiental

\section{GESTIÓN DE PROYECTOS}

La Guía del PMBOK, lanzado en 1969, el Project Management Institute (PMI), y hoy en 2016 la quinta edición, se utiliza en varias áreas con las recomendaciones de gestión de proyectos para optimizar resultados. Los proyectos y su gestión se realizan en un entorno más amplio que el propio proyecto. La comprensión de este contexto ayuda a asegurar que el trabajo se lleve a cabo de acuerdo con los objetivos, que sea gestionado de acuerdo con los procedimientos establecidos por la organización. Procesos y procedimientos para llevar a cabo el plan del proyecto de la organización, incluyen el siguiente ciclo de vida del proyecto: inicio, planificación, monitoreo y control, y cierre (PMI, 2013). Un conjunto de acciones que involucra los procesos del ciclo de vida del proyecto como fue utilizado para la gestión de actividades de la licencia ambiental (Nascimento, 2015).

Procesos de Iniciación: es la fase inicial del proyecto, cuando se identifica una necesidad particular y se traduce en un problema. El proceso de licenciamiento ambiental se inicia formalmente con la carta de concesión de licencias.

Los procesos de planificación: son procesos donde los objetivos del proyecto son refinados y detallados y se identifican y seleccionan las acciones encaminadas a la consecución de los objetivos del proyecto. En este grupo de procesos se hallan los detalles de las actividades, sus estimativas de duración y la secuencia lógica, identificación de los recursos necesarios para llevar a cabo el trabajo, el desarrollo de la programación física y financiera, así como planes de acción para facilitar la gestión de proyectos de licencia ambiental. 
Monitoreo y Control del Proceso: conjunto de procesos para asegurar que el trabajo realizado siga el plan original mediante la identificación de puntos de acciones preventivas y correctivas que eliminen las anomalías identificadas mediante la medición y el monitoreo periódico de las acciones.

Proceso de cierre: un conjunto de procesos para caracterizar el cierre formal y organizado de un proyecto mediante la evaluación de los trabajos realizados, archivo documental de los trabajos y análisis de la gestión de proyectos de aprendizaje adquirido.

La Guía de Gestión de Proyectos (PMBOK) proporciona las principales referencias para la gestión de proyectos. Para tanto, mapea las áreas de conocimiento necesario para lograr este fin y desarrolla un capítulo a cada una de estas áreas. Ellos son: Gestión de la Integración, Alcance, Tiempo, Costo, Calidad, Recursos Humanos, Comunicaciones, Riesgo, Adquisiciones y grupos de interés (PMI, 2013).

\section{CASO DE APLICACIÓN}

El uso de las principales referencias para la gestión del proyecto, de acuerdo con la Guía de Gestión de Proyectos (PMBOK) (PMI, 2013), se ha preparado y presentado siguiendo las metodologías de aplicación de buenas prácticas en la gestión de proyectos para la licencia ambiental. Siendo estos: Gestión de la Integración, grupos de interés, alcance, tiempo, calidad, costo y comunicación. Estos no serán tratados en este trabajo de Gestión de Riesgos, Contratación y Recursos Humanos, por el hecho de que no presentan una relación directa con la gestión de proyectos de concesión de licencias ambientales.

Los siguientes diagramas presentan la propuesta de integración entre las licencias ambientales y gestión de proyectos, que se implementarán en la ciudad de Río de Janeiro (Brasil).

La gestión de la integración incluye las actividades para el proceso de desarrollo de la carta de otorgamiento de licencias ambientales. Así que, para empezar el proceso, se requerirá la descripción básica del proyecto. Los documentos generados en este proceso son: el requisito de concesión de licencias y la carta de apertura. La figura 2 muestra el diagrama de flujo del proceso de gestión de la integración.

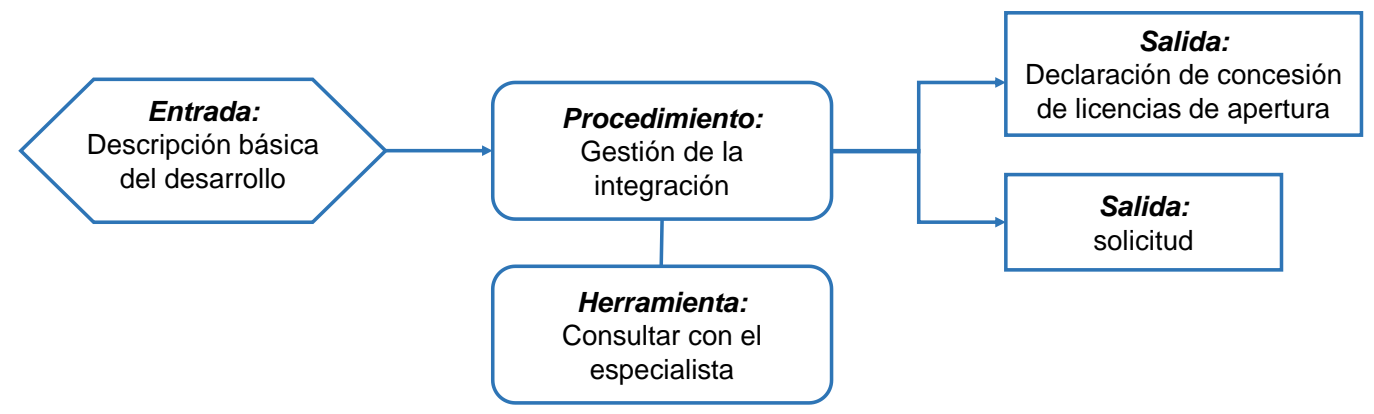

Fig. 2: Diagrama de flujo de la integración de la administración.

La gestión de los grupos de interés incluye actividades para identificar las partes interesadas, tales como la carta de apertura de licencias y la solicitud protocolada en el órgano ambiental. Las herramientas utilizadas en este paso serán las reuniones y los análisis de los interesados. El documento generado en este proceso es el registro de las partes interesadas. La figura 3 muestra el diagrama de flujo del proceso de gestión de los interesados.

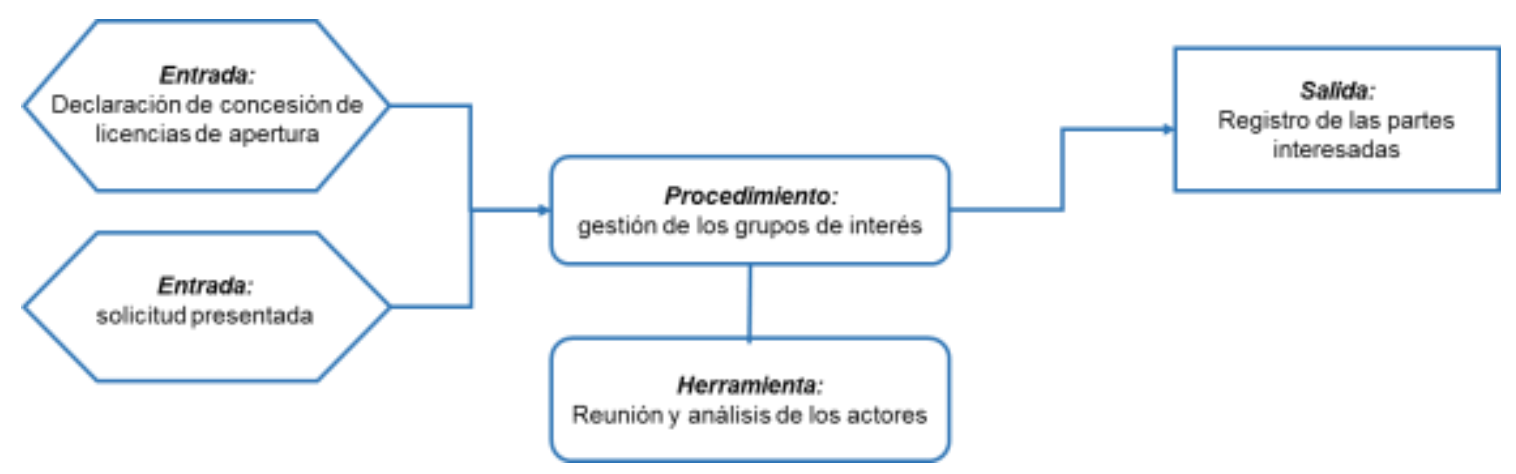

Fig. 3: Diagrama de flujo de la gestión de los interesados. 
Para la Gestión de la Evaluación de Impacto Ambiental - EIA / RIMA, la administración de ámbito incluye actividades para planificar y desarrollar el marco analítico de la EIA / RIMA. Para iniciar el proceso de planificación, será necesario, el término de referencia emitido por el órgano ambiental y el registro de las partes interesadas. La herramienta necesaria para lograr el objetivo será la reunión con expertos. El documento generado de este proceso es la especificación del alcance de la EIA / RIMA.

En la Gestión de Proyectos, el término EAP es sinónimo de diseño de la estructura analítica, una característica que pretende dividir el proyecto en partes más pequeñas (también llamadas tareas o paquetes de trabajo) y, así, más fácil de ser entendido y manejado.

Para desarrollar el marco analítico de la EIA / RIMA (EAP), inicialmente será necesario tener el alcance de la memoria para el EIA / RIMA. La herramienta usada será la descomposición de los documentos de trabajo específico. Los productos serán la línea de base de la EIA / RIMA, la estructura analítica de la EIA / RIMA (EAP) y el diccionario de estructura de analítico (EAP). La figura 4 muestra el diagrama de flujo del proceso de gestión del alcance.

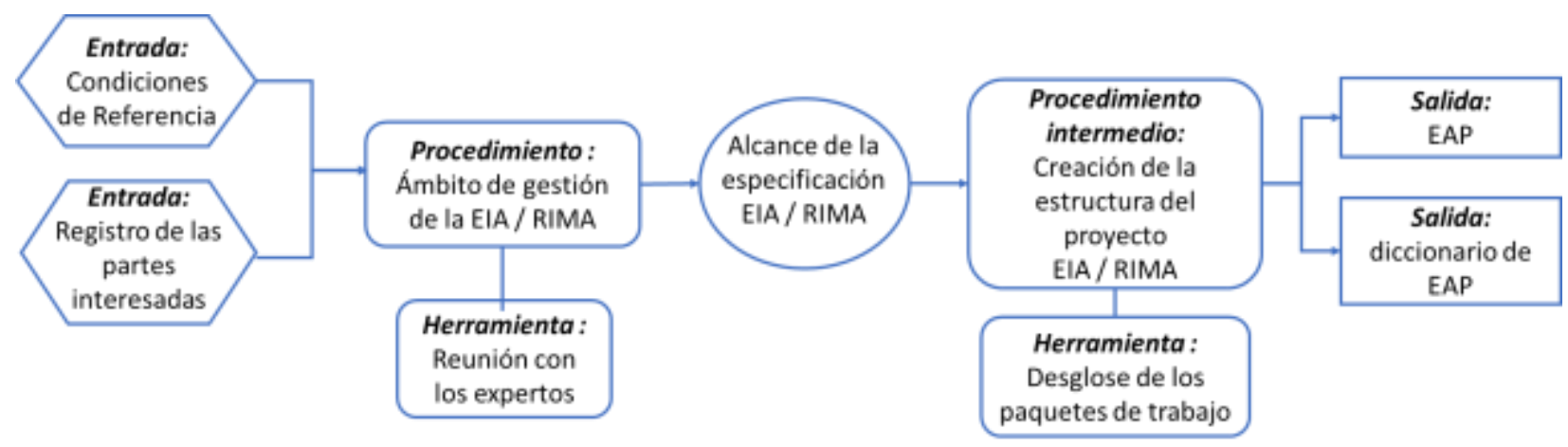

Fig. 4: Diagrama de flujo de gestión del alcance

Para la gestión de tiempo se incluye actividades para desarrollar el programa. Así que, para empezar el proceso, se va a requerir la PEA, el diccionario de la EDT, la lista de actividades, la estimación de los plazos, el calendario de los recursos, el registro de los riesgos y los costos estimados. Las herramientas utilizadas en este paso serán las reuniones con expertos y software de planificación. Los documentos generados en este proceso son: la programación física, informe de las premisas de lo previsto, histograma, lista de servicios externos y programación financiera. La figura 5 muestra el diagrama de flujo del proceso de gestión del tiempo.

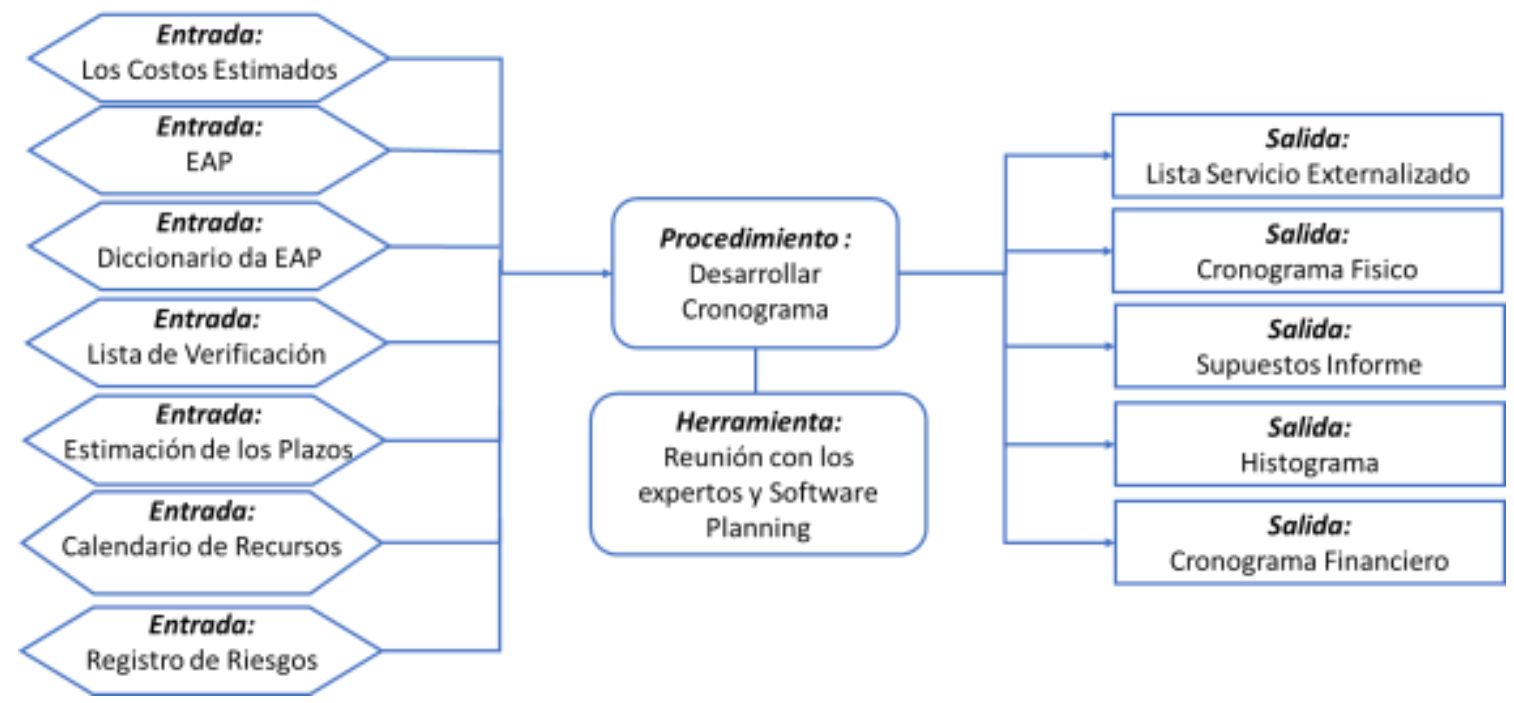

Fig. 5: Diagrama de flujo calendario de desarrollo

Para la gestión de calidad se incluye las actividades necesarias para planificar la gestión de calidad de la EIA / RIMA. Así que, para empezar el proceso se requiere: el registro de las partes interesadas, el registro de riesgos y EAP de la EIA / RIMA. La herramienta utilizada es el software de gestión de documentos. EI documento generado será el plan de gestión de la calidad. La figura 6 muestra el diagrama de flujo del proceso de gestión de la calidad. 
Para la administración de costos se incluye las actividades necesarias para planificar la gestión de los costos EIA/RIMA. Así que, para iniciar el proceso, se necesita: el cronograma financiero, la lista de servicios externalizados y el histograma de los profesionales. La herramienta utilizada es la DFC (Costo Declaración de Formación), que tiene por objeto registrar los desembolsos de la compañía, clasifica las facturas de gastos, costos y gastos. El documento generado será el plan de gestión de costos. La figura 7 muestra el diagrama de flujo del proceso de gestión de costes.

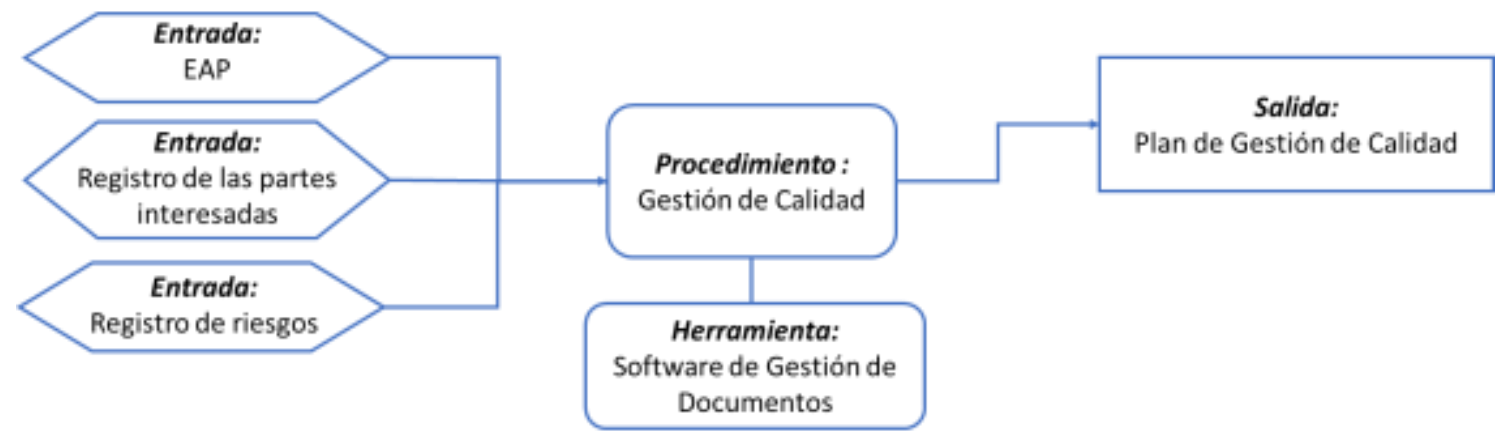

Fig. 6: Diagrama de flujo de gestión de calidad

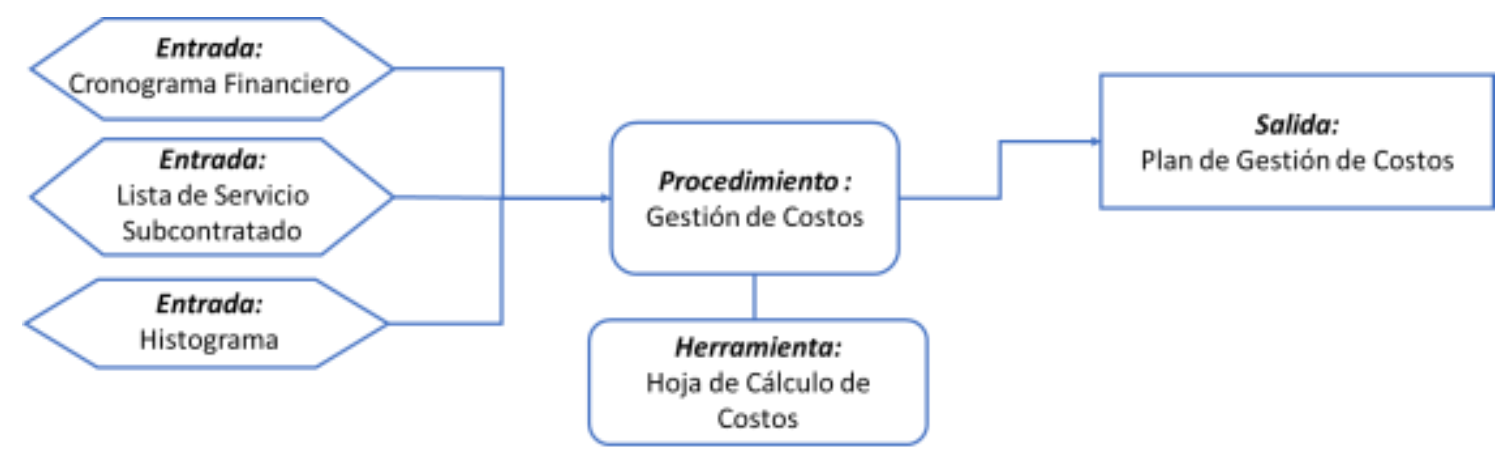

Fig. 7: Diagrama de flujo de los costes de gestión.

Para el manejo de las comunicaciones se incluye las actividades necesarias para planificar la gestión de las comunicaciones del personal y las partes interesadas. Así que, para empezar el proceso, debe haber el organigrama, la matriz de responsabilidad y el registro de los interesados. Reuniones se llevarán a cabo para la alineación de acciones de planificación, control, informes de ejecución, plan de acción y resoluciones. El documento generado en este proceso será plan la gestión de comunicaciones. Este plan debe ponerse a disposición de las partes interesadas. El profesional encargado de realizar este tipo de reuniones deberá tener el perfil integrador. Figura 8 muestra el diagrama de flujo del proceso de gestión de las comunicaciones.

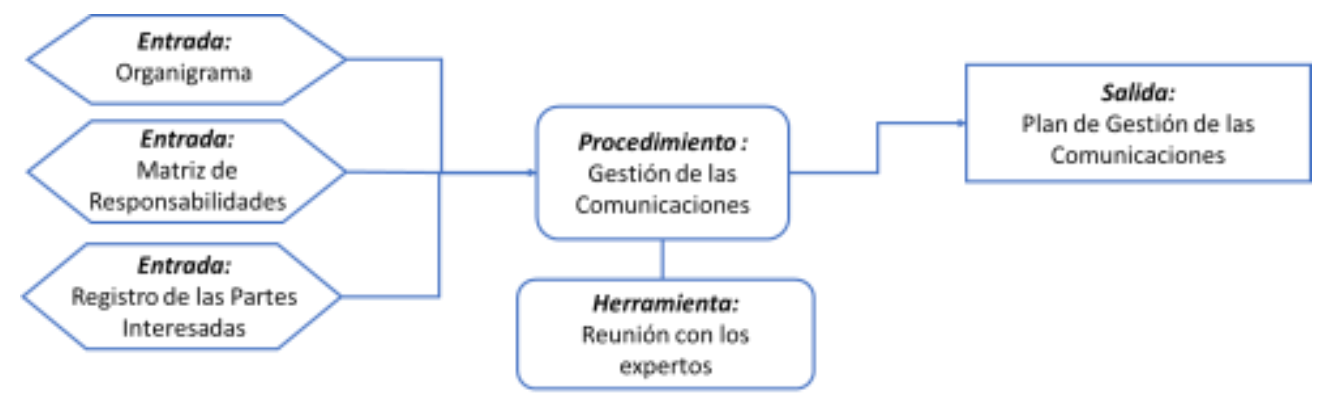

Fig. 8: Diagrama de flujo de gestión de las comunicaciones

La gestión de la integración incluye las actividades necesarias para desarrollar el plan de gestión de la EIA/RIMA. Así que, para empezar el proceso, será necesario el cronograma, plan de gestión de calidad, gestión de costos y gestión de comunicaciones. Reuniones se llevarán a cabo para integrar todas las salidas de los procesos de gestión y verificación de la interface. El documento generado en este proceso será el plan de gestión de la EIA / RIMA y será la entrada para todos los procesos de control y monitoreo. Figura 9 muestra el diagrama de flujo de la integración de la gestión. 


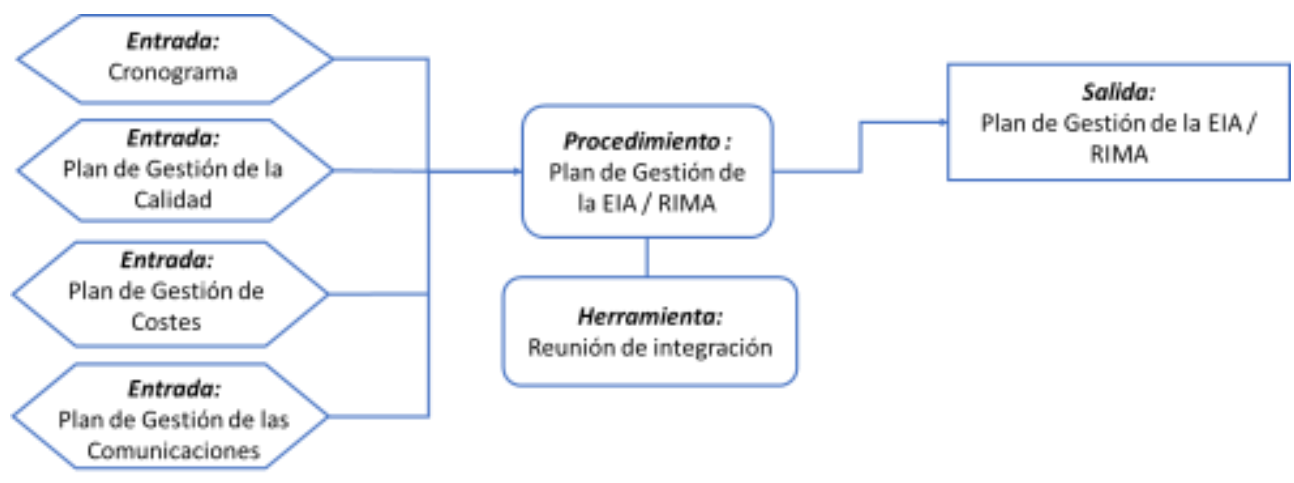

Fig. 9: Diagrama de flujo Plan de Gestión de la EIA / RIMA

Ejecución, seguimiento y control de las actividades de la EIA/RIMA incluye las actividades necesarias para ejecutar, controlar y supervisar la programación. Así que, para empezar el proceso, será necesario actualizar las actividades semanales del cronograma y el plan de gestión de la EIA/RIMA. Se llevarán a cabo reuniones para analizar el rendimiento horario, ruta crítica y las desviaciones con el apoyo de un software de gestión. Los documentos generados en este proceso son: el rendimiento de la memoria de actividades, el plan de acción y el informe de solicitud de cambio del plan de gestión de proyectos. Figura 10 muestra el diagrama de flujo del proceso de aplicación, control y monitoreo de tiempo.

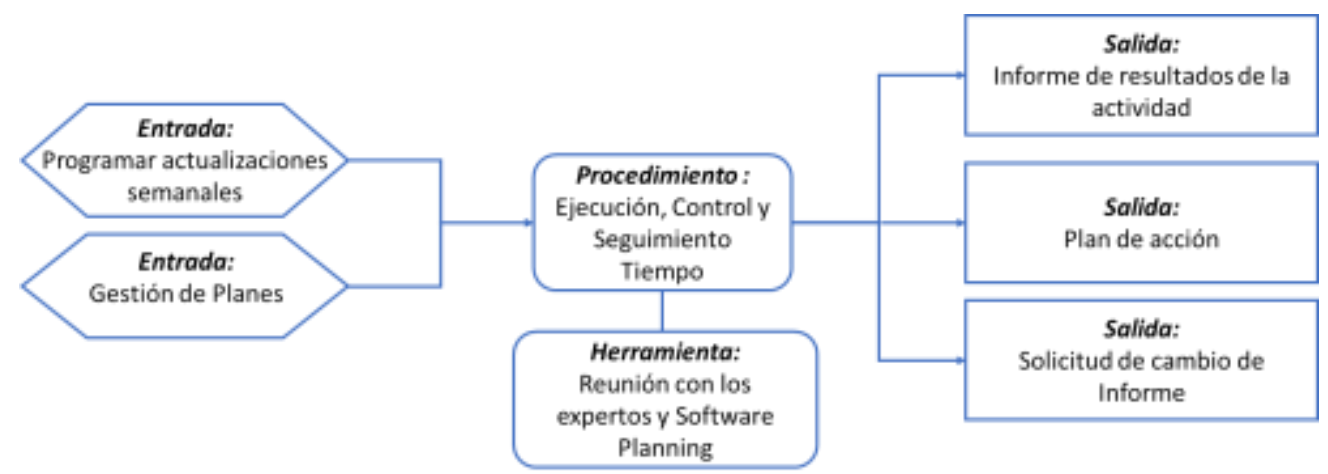

Fig. 10: Ejecución, control y seguimiento de la hora de la EIA / RIMA.

Ejecución, Control y Monitoreo de Calidad incluye las actividades para ejecutar, controlar y supervisar la calidad del EIA / RIMA. Para iniciar el proceso, serán necesarias las entregas del plan de gestión de la EIA / RIMA. Se hará un cheklist de entregas parciales a la EIA/RIMA. Los documentos generados son: informe del desempeño de calidad, informe de solicitud de cambio y el registro de las lecciones aprendidas. La Figura 11 muestra el diagrama de flujo de proceso y ejecución, control y monitoreo de calidad.

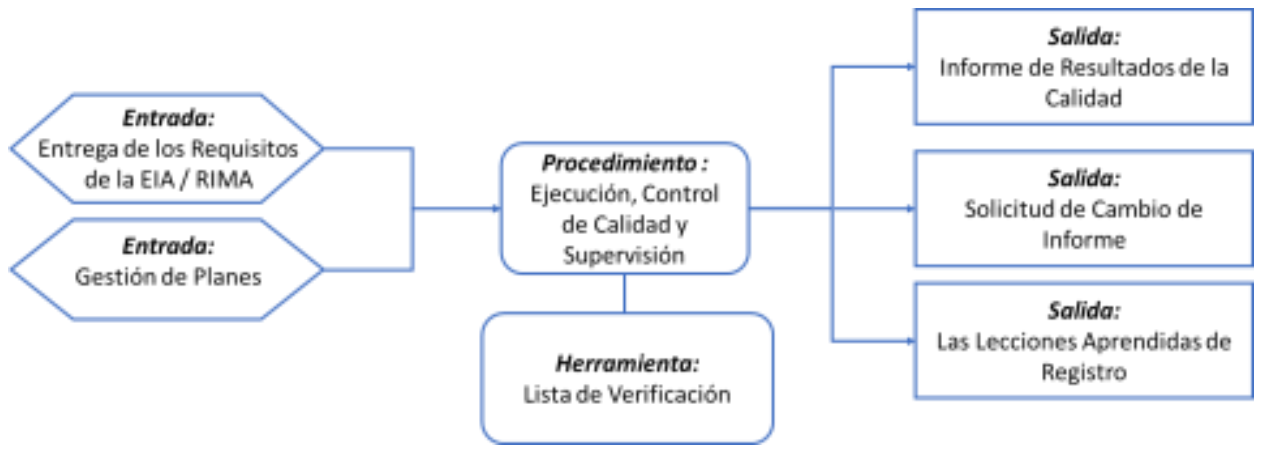

Fig. 11: Ejecución, control y monitoreo de calidad de la EIA / RIMA

Ejecución, Monitoreo y Control de Costos incluye las actividades a ejecutar, controlar y supervisar el plan de gestión de los costes EIA/RIMA. Así que, para iniciar el proceso, se requieren los siguientes documentos: el programa financiero, la lista de servicios externalizados y el histograma de profesionales. Se llevarán a cabo reuniones para analizar el rendimiento financiero y los costes adicionales con apoyo de un software de gestión. Los documentos generados en este proceso son: el informe de ejecución financiera y el plan de solicitud de cambio de dirección de proyectos. La figura 12 muestra el diagrama de flujo de los costos del proceso de implementación, control y seguimiento. 


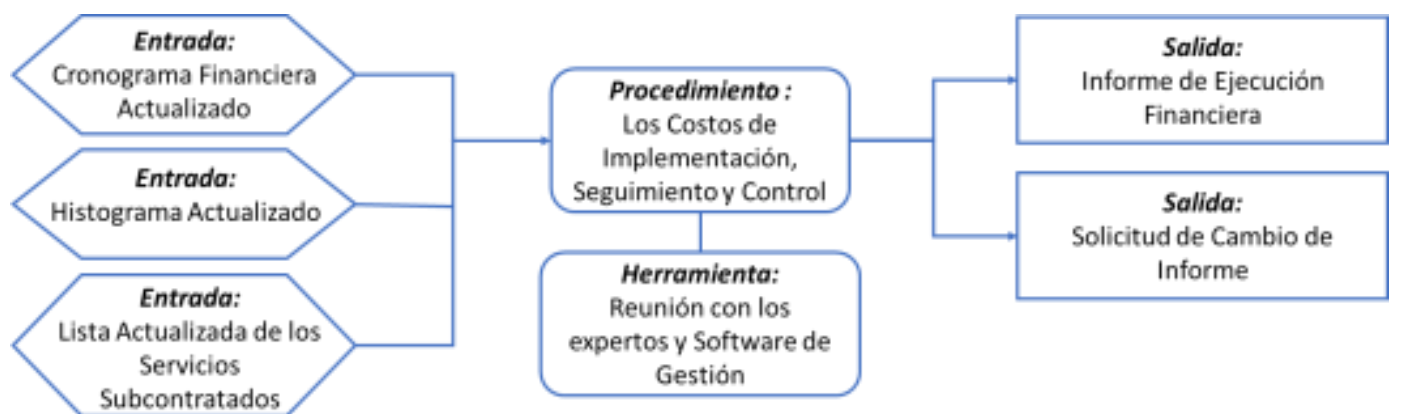

Fig. 12: Ejecución, control y seguimiento de los costos de la EIA / RIMA.

Ejecución, Control y Seguimiento de las comunicaciones incluye las actividades necesarias para controlar y supervisar el equipo de comunicaciones y las partes interesadas. Así que, para iniciar el proceso, se requiere el plan de gestión de la EIA/RIMA. Reuniones se llevarán a cabo para la alineación de las interfaces de actividades. El documento generado en este proceso será el informe de desempeño del plan de comunicación. La figura 13 muestra el diagrama de flujo del proceso de implementación, el control y la vigilancia de las comunicaciones.

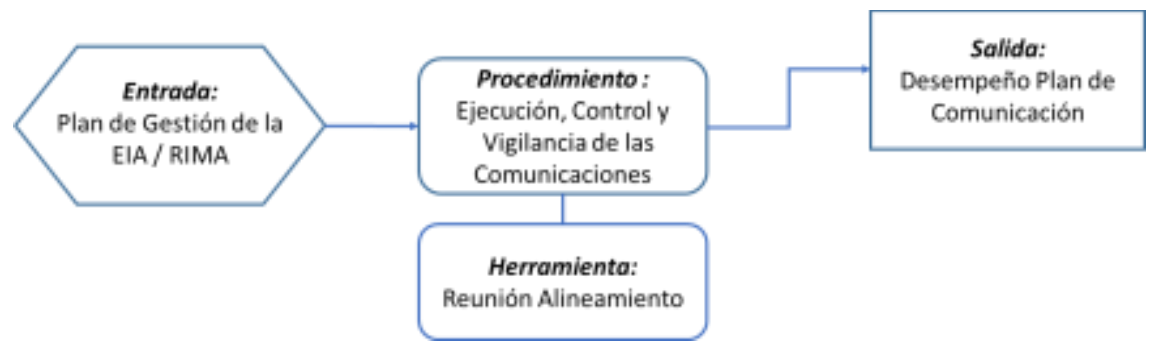

Fig. 13: Ejecución, control y seguimiento de las comunicaciones EIA / RIMA.

Ejecución, Seguimiento e Integración de Control, incluye actividades a ejecutar, controlar y supervisar la integración de las entregas de la EIA/RIMA. El plan de manejo actualizado y el informe de validación interna del EIA / RIMA. Así que para empezar el proceso se requieren: informes sobre el rendimiento de calidad, informe de desempeño de los costos, informe de ejecución de las actividades e informe del dessempeño de comunicaciones. La reunión de integración es la principal herramienta para poner en práctica este proceso. Reuniones se llevarán a cabo para integrar todas las salidas de los procesos de control y monitoreo. Los documentos generados son: el plan de manejo actualizado y el informe de validación de EIA/RIMA. Por lo tanto, después de esta etapa de estudio se presentará a la agencia ambiental para su aprobación. Si el dictamen es positivo, será enviado a LI. Si el dictamen es negativo: planificación, control y monitoreo deben ser revisados. La figura 14 muestra el diagrama de flujo del proceso de implementación, control y monitoreo de la integración.

El cierre de actividades de desarrollo y aprobación de EIA/RIMA incluye las actividades necesarias para concluir las entregas de la EIA/RIMA. Así que, para comenzar estas actividades, se requiere el parecer positivo de la agencia ambiental del EIA / RIMA. Para seguir este paso es necesario que el personal tenga comunicación diaria con la agencia ambiental para cumplir con la fecha límite. Los documentos generados en esta etapa son: el parecer positivo y la carta del proyecto (TAP). Después de este paso, se obtiene la LI y la LO. La figura 15 muestra el diagrama de flujo del proceso de cierre de preparación y aprobación del EIA / RIMA.

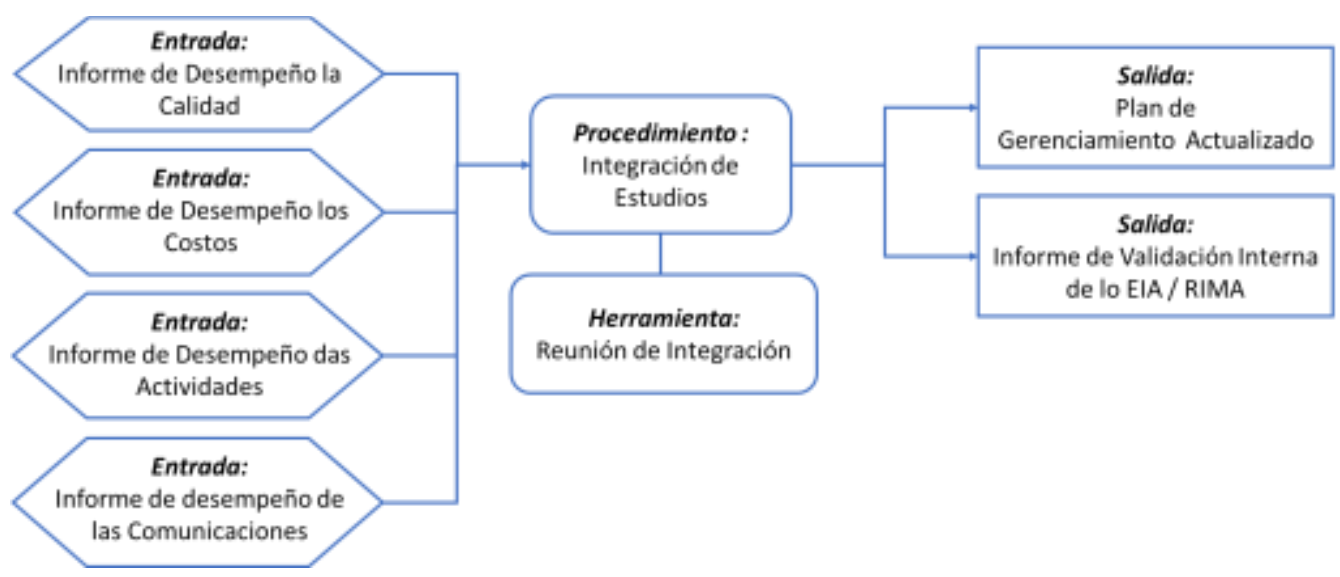

Fig. 14: La ejecución, el control y el seguimiento de la integración de la EIA / RIMA 


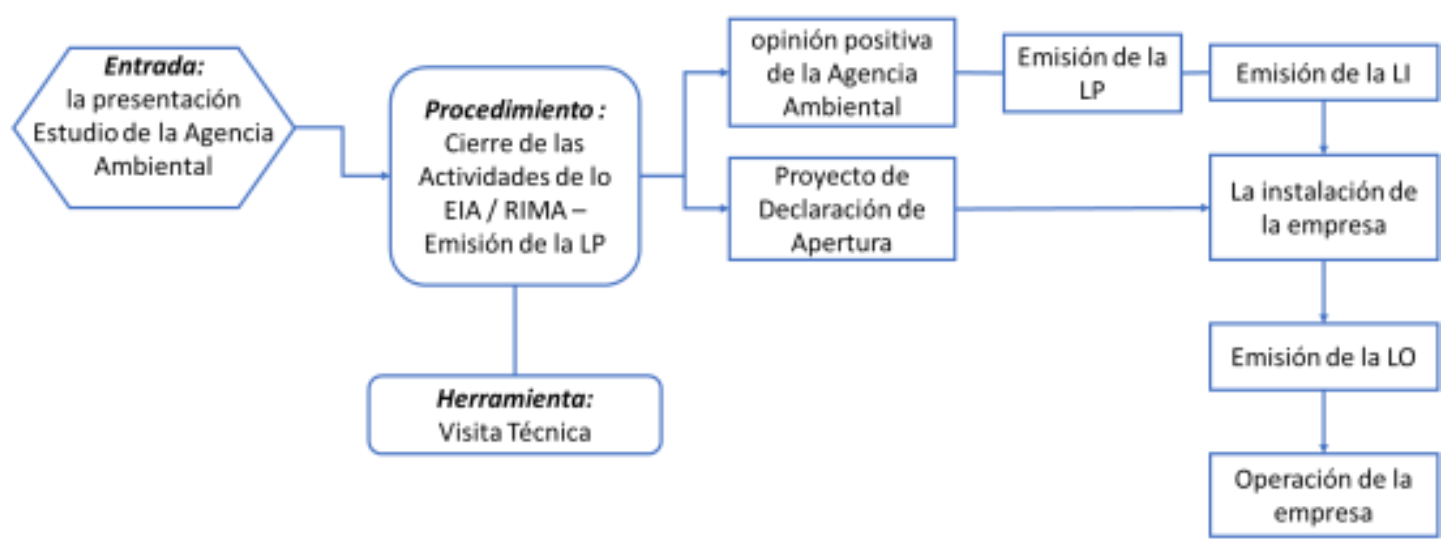

Fig. 15: El cierre de las actividades de la EIA / RIMA y la emisión de LP

Una vez aprobado el EIA / RIMA por el IBAMA y por otras autoridades ambientales pertinentes, se emitirá la LO estableciendo las condiciones de caducidad y los términos correspondientes a vista del empresario para las correcciones necesarias de sus actividades para satisfacer las exigencias impuestas por la normativa de IBAMA y de otras autoridades ambientales pertinentes. El documento aprobado por la agencia incluyen las multas previstas por la legislación que podrán aplicarse a la entidad comprometida y los casos de extinción debido a la situación de incumplimiento de las obligaciones acordadas en él, contienen también, todos los procedimientos que deben seguirse para la concesión de licencia ambiental federal del proyecto(Nascimento, 2015).

\section{CONCLUSIONES}

El proceso de licenciamiento ambiental en Brasil es complejo y largo, dependiendo del proyecto, la agencia ambiental municipal, estatal y federal. De esta manera, podemos tener una variación en la cantidad de pasos, detalle que merece la atención de modo que no sea un problema que dificulte la ejecución del proyecto.

En el EIA/RIMA el plan de manejo debe ser un procedimiento de actualización y de control de la programación física y financiera. El cronograma debe ser detallado en un nivel de funcionamiento, deberá tener un apoyo semanal y tener como principales actividades el cronograma que debe ser el espejo de la EDT, facilitando el monitoreo de la entrega de los paquetes de trabajo, estudios ambientales.

Después de publicada la LP, los retos son: mantenimiento de la licencia con monitoreo de los programas ambientales y la obtención de las licencias de instalación y operación. La interacción con la agencia ambiental es esencial para la acción correctiva, caso sean identificadas inconformidades. La inversión en esta fase es alta, con costos en mano de obra especializada.

En cuanto a la calidad técnica de los estudios (informes ambientales preliminares y estudios detallados de impacto ambiental), la buena práctica sugiere que los términos de referencia se centren en los problemas de los actores interesados, que deben participar de la formulación y pertinencias para tomar la decisión. La elaboración de los estudios objetivos y bien coordinadas, debe acoger la interdisciplinariedad, y además de criterios de revisión objetivos y paneles de revisión independientes; la mitigación de los impactos negativos y planes de monitoreo de eficiencia probada.

La integración de las herramientas de gestión ambiental y la gestión de proyectos es beneficioso para los profesionales que trabajan en el área y para los futuros profesionales. Luego, de esta forma, contribuye al desarrollo sostenible, con equipos más comprometidos con la responsabilidad ambiental presente en su proyecto de planes de trabajo.

\section{REFERENCIAS}

BIRF, Banco Internacional de Reconstrucción y Desarrollo, Licencia Ambiental para proyectos hidroeléctricos en Brasil, Brasilia, Banco Mundial (2008)

CONAMA. Consejo Nacional del Medio Ambiente Brasilia, Brasil (2015). En línea: https://goo.gl/qcypfF, acceso: 15 de septiembre (2015)

Constitución de 1988, Constitución de la República Federativa de Brasil, Brasilia, Brasil (1988). En línea: https://goo.gl/GWuSKa, acceso: 15 de septiembre (2015) 
Decreto 1753 de 3 de agosto 1994, por el cual se ordenan los títulos VIII y XII de la Ley 99 de 1993 Licencias Ambientales. Bogotá, Colombia, La Presidencia (1994)

Decreto №42050 de 25 de septiembre de 2009. Regula el procedimiento de concesión de licencias de descentralización del medioambiente mediante la celebración de acuerdos con los municipios en el estado de Río de Janeiro, y otras medidas. Río de Janeiro, Rio de Janeiro. En línea: https://goo.gl/Mh0rt4, acceso: 15 de septiembre (2015)

Decreto №8437 de 22 de abril de 2015, Regula lo dispuesto en el art. 7, caput, fracción XIV, letra "h", y el párrafo único de la Ley Complementaria №140, de 8 de diciembre de 2011, para establecer los tipos de proyectos y actividades cuya licencia ambiental será competencia de la Unión, Brasília, Brasil (2015)

Decreto №99.274 del 06 de junio de 1990, Reglamenta la Ley № 6.902, de 27 de abril de 1981, y la Ley № 6938 de 31 de agosto de 1981, que versan, respectivamente, sobre la creación de Estaciones Ecológicas y Áreas de protección del medioambiente y de la política nacional ambiental y otras medidas, Brasília, Brasil (1990)

Decreto №40.722 del 08 octubre de 2015. Regula el trámite para el sistema de licencia ambiental municipal RIO SLAM y otras medidas. Río de Janeiro. En línea: https://goo.gl/b6PCcZ, acceso: 15 de septiembre (2015)

Decreto №42.440 del 30 de abril de 2010. Modificación del Decreto 42050 de 25 de septiembre de 2009, que regula el procedimiento de concesión de licencias de descentralización del medioambiente mediante la celebración de acuerdos con los municipios del estado de Río de Janeiro. Río de Janeiro. En línea: https://goo.gl/bMkYos, acceso: 15 de septiembre (2015)

Decreto №44820 del 02 de junio de 2014. Regula el Sistema de Licencias Ambientales - SLAM y otras medidas. Río de Janeiro, Río de Janeiro. En línea: http://alerjln1.alerj.rj.gov.br/CONTLEI.NSF, acceso: 15 de septiembre (2015)

Decreto-Ley $\mathrm{N}^{\circ} 134$ del 16 de junio de 1975 . Prevé la prevención y control de la contaminación ambiental en el estado de Río de Janeiro, y otras medidas. Río de Janeiro, Río de Janeiro (1975)

Garzón, M. O., Las Licencias Ambientales y sus efectos en el ambiente, Revista de la Facultad Nacional de Salud Pública, 24 (1), 98-107 (2006)

Juras, I. A. G.M., Consultor del Legislativo XI Área de Medio Ambiente y la Ley del Medio Ambiente, Ordenación del Territorio y Desarrollo Regional y Urbano. Brasília, Brasil (2013)

Ley 19300, Aprueba bases generales del medioambiente, párrafo 2, Chile, La Presidencia (1994)

Ley 99, 22 de diciembre 1993, por la cual se crea el Ministerio del Medioambiente, el sector público reordena el Encargado de Gestión y Conservación del Medio Ambiente y los recursos naturales renovables, organiza el Sistema Nacional Ambiental, SINA y otras disposiciones, Bogotá, Colombia (1993)

Ley Complementaria №140 del 8 de enero de 2011. Brasilia, Brasil (2011)

Ley $\mathrm{N}^{\circ} 6514$ del 22 de julio de 2008. Regula las infracciones y sanciones administrativas para el medioambiente, establece el proceso administrativo federal para la investigación de esos delitos, y otros asuntos. Brasília, Brasil (2008)

Ley N6938 del 31de agosto de 1981. Dispone sobre la Política Nacional del Ambiente. Brasilia, Brasil (1981)

Ley №7804 de 18 de julio de 1989. Los cambios en la Ley №6938 del 31 de agosto de 1981, donde se establece la Política Nacional del Medioambiente, sus propósitos y mecanismos de formulación y aplicación de la Ley № 7735 de 22 de febrero de 1989, la Ley № 6.803, de 2 de julio de 1980, y otras medidas. Brasília, Brasil (1989)

Ley N ${ }^{\circ} 997$ de 31 de mayo de 1976. Proporciona el control de la contaminación del medio ambiente. Estado de São Paulo. São Paulo, São Paulo. En línea: https://goo.gl/iOiKHM, acceso: 10 de enero (2017)

Ley №3.467, de 14 de septiembre de 2000. Regula las sanciones administrativas derivadas de la conducta perjudicial para el medioambiente en el estado de Río de Janeiro, y otras medidas. Río de Janeiro, Rio de Janeiro. En línea: http://alerjln1.alerj.rj.gov.br/CONTLEI.NSF, acceso: 15 de septiembre (2015) 
Ley №9.605, de 12 de febrero de 1998. Dispone sobre las sanciones penales y administrativas derivadas de las conductas y actividades dañinas para el medioambiente, y otras disposiciones. Brasília, Brasil (1998)

Matos, J. F., Gestión de Sitios Contaminados - Un estudio de caso, Proyecto Fin de Carrera, Dept. Eng. Civil, CEFET-RJ, Rio de Janeiro, Brasil (2015)

MMA, Ministerio de Medio Ambiente, Licenciamiento Ambiental Notebook, Programa de Captación Nacional de Gestores Ambientales, Brasilia, Brasil (2009)

Nacimiento, D. R., Aplicación de buenas prácticas de gestión de proyectos en el proceso de licenciamiento ambiental, Tesis de especialización en Dirección y Gestión de Proyectos, NPPG / POLI-UFRJ, Rio de Janeiro (2015)

Nakamura, Julia Noami, El Derecho Ambiental Internacional: Construcción y Efectividad en Defensa del Medioambiente, Monografía del Departamento de Derecho, PUC-RJ, Río de Janeiro (2013)

ONU, Organización de las Naciones Unidas, Manhattan, Nueva York (2012)

PMI, Project Management Institute, Inc., Un Guía para la Gestión de Proyectos (PMBOK), 5aㅡ Edición, Pennsylvania, EE.UU. (2013)

Resolución CONEMA N ${ }^{\circ} 42$ de 17 de agosto de 2012. Consejo Estatal del Medio Ambiente. Río de Janeiro, Río de Janeiro. En línea: https://goo.gl/Uflyaf, acceso: 15 de septiembre (2015)

Romero, J., Un mundo con licencia o licencioso: el licenciamiento y la protección administrativa del ambiente del en Colombia, doi: http://dx.doi.org/10.17981/juridcuc.11.1.2015.8, CUC legal 11 (1), 183-208 (2015)

Verocai, lara, Panel de Notas 2, licencias ambientales en otros países. Brasília, Brasil. En línea: http://www.mma.gov.br/estruturas/DAl/_arquivos/iaraverocai2.pdf, acceso: 10 enero (2017) 